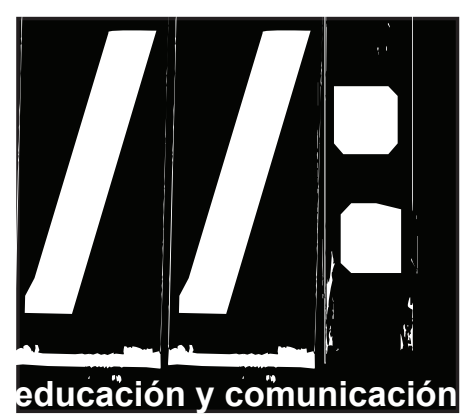

19: 35-46 Nov. 2019

\section{GCÓMO GENERAR CONGIENGIACIÓN EN LA SOCIEDAD Y EL SISTEMA EDUCATIVO COSTARRICENSE A PARTIR DE LAS CONGLUSIONES DEL DOCUMENTAL: "SUPER SIZE ME" [SÚPER ENGÓRDAME]?}

\begin{abstract}
How to generate awareness in society and the Costa Rican education system from the conclusions of the documentary: "Super Size Me"
\end{abstract}

\author{
Natin Guzmán Arce \\ Docente e investigador \\ Universidad Nacional de Costa Rica \\ E.mial: natin.guzman.arce@una.ac.cr \\ Vivian Vargas Barquero \\ Docente e investigador \\ Universidad Nacional de Costa Rica \\ E.mail: vivian.vargas.barquero@una.ac.cr
}

Resumen

Actualmente el ser humano se encuentra inmerso en una serie de actividades académicas, sociales y de esparcimiento pasivo que le dejan poco tiempo para la actividad física y la recreación física. Desafortunadamente, a nivel mundial se ha pasado de un modelo de alimentación casera a alimentos precocinados o comida rápida. Enmarcando en este panorama se encuentra la película-documental "Super Size Me" (Súper Engórdame) la cual argumenta cómo el protagonista decide someterse a un experimento socio-alimenticio de la cultura nutritiva americana basada en comida rápida y pone sobre la mesa uno de los problemas más serios de salud en Estados Unidos: la obesidad. En contraste, Costa Rica se reconoce a nivel internacional por un clima de paz, país sin ejército y su exuberante naturaleza con sus cientos de hectáreas protegidas. Sin embargo, los índices de obesidad van en aumento lo que ha llevado a que surjan esfuerzos de parte del gobierno costarricense por enfrentar dicha epidemia desde las aulas y los comedores escolares.

Palabras clave: educación alimenticia, obesidad, salud, aprendizaje

https://doi.org/10.25267/Hachetetepe.2019.v2.i19.5 


\title{
II: Salud, Educación y Medios de Comunicación
}

\begin{abstract}
Nowadays humans are immersed in academic, social and passive leisure activities that leave little room for physical recreation and workout. Unfortunately, worldwide society has gone from a homemade cooking model to a precooked food or/and fast food. To illustrate this phenomenon, the documentary "Super-Size Me" depicts the main protagonist as an individual who conducts a socio-food experiment of the American food culture based on fast food and puts on the table one of the most serious health problems in the United States: obesity. In contrast, Costa Rica is recognized as an international level for a climate of peace, a country without no army and its exuberant nature with its hundreds of hectares protected. However, obesity rates are increasing, which has led to the emergence of efforts by the Costa Rican government to address this epidemic from classrooms and school cafeterias.

Key words: nutrition, education, obesity, health, learning
\end{abstract}

Recibido 29-09-2019 / Revisado 13-10-2019 / Aceptado 15-10-2019 / Publicado 01-11-2019 


\section{Introducción}

$\mathrm{L}^{2}$ a famosa frase que reza que "somos lo que comemos" de Ludwig Feuerbach, filósofo y antropólogo alemán, ha tenido mayor relevancia a partir de los inicios del siglo XXI. Feuerbach (1850) en su ensayo "Enseñanza de la alimentación" (Lehre der Nahrungsmittel: Für das Volk), escribió: "si se quiere mejorar al pueblo, en vez de discursos contra los pecados denle mejores alimentos. El hombre es lo que come". Si se quiere, a Feuerbach, puede verse como un visionario del mundo moderno. $\mathrm{Su}$ época movilizada por la revolución industrial dictaba una serie de cambios de una sociedad, cuya economía estaba basaba en la agricultura, a una competencia sociopolítica dictaba por la ciencia, la tecnología y el comercio. Lejos estaba Feuerbach de prever las sociedades futuras especialmente aquellas que a través de la globalización han llegado a la cuarta revolución (Blázquez, 2016: 12).

En la actualidad el ser humano se encuentra inmerso en una serie de actividades académicas, sociales y de esparcimiento pasivo que le dejan poco tiempo para la actividad física y la recreación física. Al igual que la revolución industrial, la cuarta revolución (Blázquez, 2016: 12) requiere de un nuevo tipo de trabajador más automatizado y capacitado en la nube o date base. El colaborador de las empresas en el siglo XXI se encuentra cada día ligado al ordenador/computador con menos tiempo para socializar, distraerse y cuidar de su alimentación diaria. Este último punto es sumamente importante, ya que en la era actual se ha pasado de un modelo de alimentación casera a alimentos precocinados o, aún más sencillo, a los restaurantes de comidas rápidas donde se combina una preparación expedita, poco tiempo de espera y precios asequibles al presupuesto del trabajador de la nueva revolución industrial conocida como 4.0 que busca una asignación más eficiente de los recursos dando prioridad al uso de herramientas tecnológicas en vez del recurso humano (CyC Prisma, 2016: 14).

Enmarcado en este panorama se encuentra la películadocumental "Super Size Me" (Súper Engórdame) del cineasta Morgan Spurlock (2004). Spurlock, quien se graduó en la Escuela de Artes de la New York conocida como Tisch School of Arts, inspiró su guion después de ver en los noticieros a dos adolescentes que demandaban a la corporación McDonald's por los daños en la salud provocados por la comida que ofrecen. El argumento de este documental se desarrolla cuando Morgan Spurlock (2004), quien es director y personaje principal, decide someterse a un experimento socio-alimenticio de la cultura nutricional americana que estimulada por el bombardeo de anuncios comerciales de comida basura/chatarra de las cadenas de comida rápida incita a comer este tipo de alimento constantemente, y pone sobre la mesa uno de los problemas más serios de salud en Estados Unidos: la obesidad, considerado por la Organización Mundial de la Salud (OMS, 2004) como una epidemia y ratificado en el estudio ejecutado en el 2015 sobre obesidad a nivel mundial que concluye que la ingesta calórica en los países con mayor poder adquisitivo se ha duplicado en los últimos 40 años (OMS, 2016: 452).

En el experimento socio-alimentario llevado por Spurlock, éste se somete por 30 días a comer solo comida basura/chatarra de la conocida cadena de restaurantes de comida rápida: McDonald's. Para este fin, el protagonista visita el restaurante y consume 


\section{II: Salud, Educación y Medios de Comunicación}

los tres tiempos principales de comida de su menú; además aprovecha las ofertas permanentes proporcionadas por la cadena de comida rápida conocidas como "agrandar el combo" esto consiste en obtener una hamburguesa o papas fritas de mayor tamaño y rellenar el refresco gaseoso las veces que se quiera. Acompañado con un equipo médico y nutricional, se midieron los cambios físicos, químicos y emocionales causados por este tipo de dieta. Los resultados al final del experimento fueron sorprendentes y congruentes. Spurlock había consumido un aproximado de 5.000 calorías diarias, aumentó 24.5 libras (11.113 kg), su grasa corporal pasó de $11 \%$ a $18 \%$, mostró señales de depresión, tuvo pérdidas de libido y en el laboratorio sus niveles de colesterol y triglicéridos aumentaron significativamente (Pintor et al, 2017: 5-6).

Este tipo de experimento humano donde personas sanas se sometan a una dieta tan calórica son poco frecuentes, ya que son agresivos y tienen efectos colaterales complejos. Sin embargo, "Super Size Me" embozó una lista de aspectos positivos sobre lo que la OMS denominó "globisity" cuya traducción al español equivale a "globesidad" (Costa Font y Mas $\mathrm{N}$ 2014: 5). Este nuevo concepto captura el mundo desarrollado ligado a la economía de los individuos y su comportamiento socio-alimenticio. El primer aspecto está directamente relacionado con la cadena de restaurantes McDonald's que agregó a su menú los porcentajes calóricos y expandió la oferta a otras opciones de comidas más balanceadas como ensaladas y/o hamburguesas estilo gourmet y para niños fruta o yogurt en la 'cajita feliz'. Similar a este cambio, se puso sobre la palestra social el impacto negativo de no tener una dieta sana y balanceada. Otro aspecto relevante, es el poder de la publicidad sobre los individuos. La película empieza con un grupo de niños vocalizando de memoria un "jingle" publicitario que resalta el nombre de ciertas cadenas de comida rápida. Este experimento expuesto en la película "Super Size $M e$ " es, prácticamente, aplicable a toda sociedad en una $u$ otra medida especialmente en los países más occidentalizados. Por ejemplo, la sociedad costarricense no escapa a esta epidemia y en los últimos 60 años, la población de Costa Rica pasó de tener una desnutrición de más del 50\% a un crecimiento de obesidad de la ciudadanía de una forma alarmante con un índice de obesidad del 34\% (Semanario Universidad, 2019: 1). Ante tal realidad, ¿Qué acciones se han tomado en escuelas y colegios de parte del gobierno de la república para combatir esta epidemia? ¿Qué hace falta por hacer para concienciar a la población estudiantil de tener una alimentación sana y coadyuve a su crecimiento cognitivo y un aprendizaje eficaz? ¿Qué tipo de conclusiones puede sacar el pueblo de Costa Rica de una película como "Super Size Me"? Estas son algunos interrogantes que este ensayo busca explicar y contribuir para mejorar el sistema educativo costarricense a nivel integral, que incluya una dieta balanceada idónea para favorecer el aprendizaje de los estudiantes. Como educadores, se exponen estas ideas con la premisa de querer mejorar la enseñanza nutricional de los estudiantes. Sin embargo, antes de exponer esos detalles, es importante resaltar datos de la obesidad como una enfermedad que tiene ramificaciones en todas las ramas sociales incluyendo la educativa.

\section{La Obesidad: Pandemia Mundial}

La obesidad y el sobrepeso según la OMS se definen como la acumulación anormal de grasa que puede ser perjudicial para la salud. Una forma simple de me- 
dir la obesidad es medir el índice de masa corporal (IMC), esto es el peso de una persona en kilogramos divido por el cuadrado de la talla en metros. Cualquier individuo cuyo IMC sea igual o superior a 30 es considerado obeso y con uno igual o superior a 25 es considerado con sobrepeso (OMS 2012, p 14 ver cuadro 1).

Clasificación de indices de masa corporal

\begin{tabular}{|c|c|}
\hline Índice de Masa Corporal & Clasificación \\
\hline 18.5 o menos & bajo peso \\
\hline 18.5-24.9 & peso normal \\
\hline $25-29.9$ & Sobrepeso \\
\hline Mayor a 30 & Obesidad \\
\hline 30-34.9 & obesidad tipo 1 \\
\hline 35-39.9 & obesidad tipo 2 \\
\hline Mayor a 40 & obesidad mórbida \\
\hline
\end{tabular}

Cuadro 1: tomado de informe Sobrepeso y Obesidad (OMS, Salud 2010)

El cuadro 1 nos da una imagen porcentual de cómo se clasifica la obesidad con relación al IMC. Sin embargo, hay otros rubros importantes que se deben considerar en la ecuación para analizar efectos nocivos en la salud como lo son la circunferencia de la cintura (obesidad abdominal) que en hombres debe ser no mayor a $90 \mathrm{~cm}$ y en mujeres a $80 \mathrm{~cm}$. En el 2016 la OMS estimó que 1.900 millones de personas mayores de 18 años mostraban sobrepeso, y aproximadamente 650 millones podían categorizarse como obesos, casi el 13\% de la población adulta del planeta (OMS, Obesidad y Sobrepeso 2017 p.311). Definitivamente, estas cifras son alarmantes no sólo porque la obesi- dad representa un problema de sanidad pública, sino porque las consecuencias económicas para los países son muy altas.

La obesidad no es un azar del destino, sino el resultado de una sociedad globalizada cuyo desarrollo socioeconómico demanda menos actividad física y más inmovilidad en las labores diarias ligado a una dieta rica en carbohidratos, grasas saturas, azúcar y accesibilidad de los productos. En su informe "Sobrepeso y Obesidad" (OMS 2017 p. 311) se establece una relación inequívoca entre países ricos y sus niveles de obesidad en la población alcanzando también a los países en vías de desarrollo o bien países con una dieta más sana y conservadora. Un ejemplo de esto es que este informe presenta a Los Estados Unidos con el mayor número de obesos con un porentaje de 32.2, seguido de México con 30.2, el Reino Unido con 23 y un total de 27 naciones más terminando con Japón cuyo porcentaje es de 3.0 lo que indica que hay un sector de la población en Japón que también ha cambiado su dieta tradicional según el estudio (OMS 2017 p. 314).

Para muchas personas la obesidad puede entenderse como un problema de estética y de lucir bien. No obstante, la obesidad tiene secuelas de mayor magnitud a nivel social, moral, psicológico, emocional y de salud corporal. La obesidad está asociada a múltiples trastornos de salud tales como: hígado graso, diferentes tipos de cáncer (seco, páncreas, esófago, endometrio, colorrectal, riñón, y otros), enfermedades pulmonares, diabetes, problemas coronarios y cardiacos, venosa crónica, enfermedad perito dental entre algunas más. Evidentemente estás secuelas han hecho que organismos como la OMS haya declaro la obesidad como una epidemia y desde el año 2002 viene traba- 


\section{II: Salud, Educación y Medios de Comunicación}

jando en combatirla con el programa: La Estrategia Mundial sobre el Régimen Alimentario, Actividad Física y Salud que todos los países miembros aprobaron en mayo del 2004 en donde se etiquetó a la obesidad como una epidemia (OMS, 2004).

Es así como surge en los países miembros la necesidad de trabajar como gobierno en implementar lineamientos que creen conciencia y cambios en la ingesta de alimentos y dieta de sus ciudadanos. Costa Rica siendo miembro activo de la OMS toma conciencia de que también su población está siendo afectada seriamente por esta epidemia. Los lugares obesogénicos superan aquellos dedicados al ejercicio y actividad física. Según la organización de las Naciones Unidades para la Alimentación y la Agricultura (FAO siglas en inglés), Costa Rica ocupa la sexta posición en su población adulta obesa (Jiménez, 2019 p.1). Si esta cifra es preocupante, alarma más la cifra de la niñez y adolescencia costarricense donde se habla de la cantidad de 118.000 mil escolares con sobrepeso u obesidad, una generación nueva pero más enferma con los problemas de salud mencionados anteriormente. De acuerdo con la Dra. Xinia Fernández Rojas, de la Escuela de Nutrición de la Universidad de Costa Rica, la obesidad aumentó en un $13 \%$ en los últimos años (Jiménez 2019 p.1). Contrario a otros reportes donde la obesidad se asocia con poder adquisitivo como en los países ricos, en Costa Rica existe un vínculo muy intrínseco entre la obesidad y la pobreza. En el censo escolar llevado a cabo por el Ministerio de Educación Pública (MEP) sobre Peso/Talla en el 2016 (MEPcenso 2017 p.4) reveló que un 64\% se encuentra en su peso normal, $20 \%$ con sobrepeso, $14 \%$ con obesidad y un $2 \%$ con desnutrición. Si bien, las cifras tienen a ser positivas, cuando se hila un poco más fino, se denota una mayor relación entre un peso normal con un estado social más alto, mientras que los índices de sobrepeso y obesidad se encentran en escolares de comunidades más marginales y de clase social inferior. Fernández, aseguró, que obviamente los escolares no parecen estar muriendo de hambre, pero, sí es importante establecer criterios alimenticios geográficos que grafiquen la relación alimento-nutrición y peso ideal. Definitivamente, el gobierno de Costa Rica ha empezado a trabajar con relación a estos altos índices de obesidad en el país, y uno de sus frentes más fuertes es el sistema público y privado escolar.

\section{Acciones tomadas por el Gobierno de Costa Rica para combatir la obesidad en el Sistema Educa- tivo}

Costa Rica se reconoce a nivel internacional por un clima de paz, país sin ejército y su exuberante naturaleza con sus cientos de hectáreas protegidas. Sin embargo, como país en vías de desarrollo tiene problemas serios que solucionar a nivel político, social, económico, educativo y de seguridad social. Es en este último punto donde los índices de obesidad tienen su peso importante. Una sociedad físicamente enferma es una sociedad muy cara para el sistema de salud pública de cualquier nación. Con los índices de obesidad expuestos en el apartado anterior, nacen esfuerzos de parte del gobierno costarricense por enfrentar dicha epidemia. En el marco de la obesidad, se hacen esfuerzos importantes a nivel macrosocial de concienciar a la ciudadanía de mejorar sus hábitos alimenticios. Es así, como en mayo del 2019 se firma una carta de compromiso para combatir la obesidad y el sobrepeso, documento que es firmado por diez 
ministros y el presidente de la Caja Costarricense del Seguro Social (CCSS) cuyo compromiso es mejorar la alimentación pública para el año 2030 especialmente en el sector educativo.

No obstante, el MEP junto con la Universidad de Costa Rica, desde el año 2017 se dispusieron a cambiar el menú de alimentación en los centros educativos públicos y privados. Es importante recalcar que en el sistema educativo costarricense de carácter público posee comedores escolares donde los estudiantes reciben desayuno y almuerzo. Antes del 2017, el menú se basaba mayormente en carbohidratos. Con los nuevos menús establecidos en el 2017, los estudiantes comen más frutas y verduras acorde a su nivel nutricional y se aumenta el consumo de agua. Se obligó a todos los centros educativos a eliminar refrescos gaseosos y dejar solo refrescos naturales o agua. También, se eliminaron todo tipo de comidas rápidas como pizzas, hamburguesas o similares. De estos comedores escolares se benefician 800.000 mil estudiantes en un total de 4800 centros entre primaria y secundaria. En términos económicos son unos 300 millones de dólares que se invierten con una dieta más sana (Jiménez, 2019 p.5). En el gobierno de la presidenta Laura Chinchilla (2010-2014), el gobierno implementó un programa llamado Red de Cuido el cual consistía en cuidar a los niños y niñas que no tenían edad para ir a las escuelas todavía para que sus progenitores (en su mayoría jefas de hogar solas) puedan salir a trabajar. Este programa alberga unos 7.000 niños y también han modificado su menú similar al que se ofrece en los centros educativos.

Definitivamente, estas iniciativas son esfuerzos importantes para combatir la obesidad y sobrepeso en la comunidad educativa del país, empero, hay algu- nos puntos que deben ser observados. Por ejemplo, a pesar de existir un comedor escolar, muchas instituciones conceden la administración de las cafeterías a proveedores externos que venden servicios alimentarios. A pesar de que debe someterse a las regulaciones establecidas por el Ministerio de Educación Pública, la regulación diaria del menú es una labor titánica. Este tipo de establecimientos existen en instituciones educativas donde los comedores educativos se vuelven insuficientes para la población estudiantil.

Estos menús son muy importantes para colaborar en la concienciación de una alimentación sana y rica en nutrientes, pero ¿serán efectivos para el estudiante? Existe una correlación importante en el aprendizaje y la dieta que podría estar siendo obviada por políticas buenas pero masivas como éstas. Se requiere de un análisis más exhaustivo para generar planes de acción más eficaces en el conocimiento de la población estudiantil.

\section{Acciones que hacen falta en la Integración de una Dieta Sana y el Aprendizaje}

Alimentarse correctamente y en horarios adecuados tiene un impacto sustancial en el aprendizaje cognitivo de todos los seres humanos especialmente en los niños y adolescentes; así, la educación y la nutrición se refuerzan recíprocamente. Los esfuerzos en ligar una buena dieta con el aprendizaje tienen efectos positivos en los individuos. La mayoría de los estudios han demostrado que un individuo con malnutrición tiene retraso en su desarrollo físico, mental y cognitivo (Julio 1993). Haciendo referencia a la película de "Super Size Me", la comida rápida es menos nutritiva y más dañina a todo nivel para el individuo y se debe 


\section{II: Salud, Educación y Medios de Comunicación}

combatir a toda costa. La comida rápida o "chatarra" (junk food) se divide en tres grupos la callejera, que es atractiva por ser de bajo costo, pero con un gran riesgo por su manipulación siendo portadora de virus y enfermedades. El otro grupo es la comida de paquetes que tiene la característica de poseer edulcorantes, colorantes y preservantes. Por último, está la comida con calorías vacías o comidas rápidas, ricas en azúcar, grasa y carbohidratos (Jiménez 2019, p 2). El médico Julián Chaparro Romero de la Universidad La Salle, San José, lidera una campaña en contra de este tipo de alimentación y recomienda otro tipo de alimento que coadyuve al individuo en todas sus áreas. Por ejemplo, Chaparro (Jiménez 2019, p 2) recomienda que para el desayuno se tome una fruta, como la manzana rica en fibra, un lácteo y un cereal. Al almuerzo puede ser una carne blanca (pollo o pescado) verduras, arroz integral y agua. Para la cena sugiere la ingesta de pasta con alguna proteína y una crema de verduras. Este tipo de dieta según Chaparro ayuda al estudiante a tener un mayor nivel de concentración y conocimiento. La mala alimentación es un problema de salud pública y debe verse como tal. Por tanto, crear conciencia alimenticia en los estudiantes para que después estas se conviertan en hábitos diarios es imperativo.

La Universidad de Standford (2019) (bajo su apartado Nutrición: Edad Escolar sugiere lo que ha denominado "The Plate" (El Plato) para niños escolares de 6 a 12 años. Según los expertos de la universidad, durante esta etapa de la vida y del crecimiento humano, los individuos desarrollan hábitos alimenticios importantes, gustos y aberraciones. Debido a esta edad crítica, es importante establecer un menú saludable integral para su crecimiento físico, intelectual y emocional. En su programa El Plato, debe haber una porción de fru- tas, granos, vegetales, proteínas y un lácteo tal y como se muestra en el cuadro 2.



Ejemplo de "El Plato"

Cuadro 2 tomado de la organización Stanford Childrens (1)

Para ejemplificar más la gráfica, es importante desglosar el menú sugerido por el apartado nutricional expuesto por la Universidad de Stanford. El cual se basa en posibles consejos prácticos y aplicables a los alumnos en las edades mencionadas como se muestra en el siguiente cuadro 3 . 
Menú Nutricional: Universidad de Stanford (niños de 6-12 años)

\begin{tabular}{|l|l|}
\hline \multirow{5}{*}{ Desayuno } & Fruta \\
& Leche \\
& Rosca de pan \\
& Tostada con queso \\
& Cereal \\
& Sándwich de mantequilla de maní \\
\hline \multirow{4}{*}{ Alimentos en } & Fruta \\
la Escuela & Yerduras y salsa \\
& Sándwich de pavo o pollo \\
& Queso y galletas \\
& Leche y cereal \\
\hline
\end{tabular}

Cuadro 3 tomado de la organización Stanford Childrens

Los beneficios de este menú nutricional basado en lo dictado por el Departamento de Agricultura de los Estados Unidos, son bastantes positivos. La imagen de "My Plate" (Mi Plato) se clasifica en cinco clases de conjuntos de alimentos y subraya el consumo nutricional de la siguiente forma (Standford 2019):

$\square$ Granos. Alimentos que se hacen a partir de trigo, arroz, avena, harina de maíz, cebada $\mathrm{u}$ otros granos de cereales son productos de granos. Los ejemplos incluyen salvado, arroz integral y harina de avena.

$\square$ Verduras. Varíe sus verduras. Elija una variedad de verduras coloridas, incluidas verduras verde oscuro, naranjas y rojas, legumbres (arvejas y chauchas) y verduras ricas en almidón.
Frutas. Cualquier fruta o jugo con $100 \%$ de jugo de fruta cuenta como parte del grupo de frutas. Las frutas pueden ser frescas, enlatadas, estar congeladas o secas, y pueden ser enteras, estar cortadas o en puré.

Lácteos. Los productos lácteos y muchas comidas hechas a partir de leche se consideran parte de este grupo alimentario. Concéntrese en productos sin grasa o bajos en grasa, como también en los que son altos en calcio.

Proteína. Sea austero con la proteína. Elija carnes y aves magras o con poca grasa. Elija más pescado, nueces, semillas, arvejas y chauchas.

Esta distribución alimentaria debe ir acompañada de una dieta saludable y actividad física. Los resultados de someter a los hijos o estudiantes a un estilo de vida saludable son buenos ejemplos para crear individuos con conciencia nutricional y personas que sepan cómo elegir y vivir para un régimen alimentario presente en su cotidianidad.

\section{Conclusiones del Documental "Super Size Me" para la Sociedad Costarricense}

Sin duda, el documental deja una gran enseñanza sobre el descuido alimentario y negligente de los individuos al dejarse llevar por una manipulación publicitaria o bien por la desidia de ensuciar un poco sus cocinas al preparar sus alimentos justificado por la actitud de 
no tener tiempo. El mundo globalizado ha hecho que las sociedades hayan cambiado a nivel cultural, mental, social y alimenticio. En esta línea, se encuentra la población costarricense y a pesar de los esfuerzos, sí queda mucho trabajo por hacer. Las conclusiones que se derivan del documental aplicables a la comunidad costarricense se detallan a continuación.

1- Sin duda se han hecho esfuerzos importantes por parte del gobierno por empezar a combatir el problema de la obesidad y el sobrepeso creciente en los últimos años. Sin embargo, queda mucho trabajo por hacer pues las políticas parecen mayormente en el papel y no en campañas efectivas que impacten el comportamiento de los ciudadanos.

2- Los esfuerzos del Ministerio de Educación por eliminar alimentos ricos en azúcar, carbohidratos y grasas saturadas en las escuelas y colegios son positivos. No obstante, como dice la directora del área de Niñez y Adolescencia de la Defensoría de los Habitantes de Costa Rica, Kathya Rodríguez Araica (Jiménez 2019,p 3), existe un gran desafío al concluir si dichas modificaciones han tenido un impacto directo en la dieta de los estudiantes. No hay estadísticas que demuestren que tan efectivos han sido. También, explica Rodríguez, que no hay un estudio que indique que lo ofrecido por los comedores escolares sean los alimentos requeridos para el desarrollo físico e intelectual que ocupan los estudiantes.
3- A pesar de que los establecimientos de comida rápida están obligados a indicar las cantidades calóricas de sus comidas, no existe ningún tipo de regulación para delimitar la publicidad, o bien, promover una alimentación sana en la sociedad costarricense.

4- La dieta del costarricense sigue siendo muy rica en carbohidratos, pero al cambiar nuestra fuerza laboral, el sedentarismo ha hecho que la población se vea inmersa en la epidemia de la "globesidad". Sería importante, que el gobierno de la república haga campañas permanentes sobre educación física, educación nutricional e incentive un menú saludable desde los centros educativos, comités provinciales y cantonales, instituciones deportivas, universidades estatales, grupos juveniles, religiosos y geriátricos. Una campaña integral que no solo informe, pero que ayude a cambiar los hábitos de alimentación de los ticos (2).

5- Se debe incluir el tema de una dieta sana en todos los currículos educativos y como eje transversal. Desde la escuela y colegio principalmente para crear conciencia colectiva con relación a la alimentación. Cierto que es una labor difícil, pues existen intereses muy fuertes y económicos que entran en juego. Sin embargo, una sociedad saludablemente sana es una gran economía para el país.

6- Los hábitos alimenticios conforman costumbres diarias que se deben fomentar a temprana edad los cuales favorecen el estado de salud y nutrición de los consumi- 


\section{¿Cómo generar concienciación en la sociedad y el sistema educativo Costarricense a partir de las conclusiones del documental: "Super size me" (súper engórdame)?}

\section{Monográfico}

dores. Por esta razón, los comedores escolares costarricenses deben ofrecer menús variados y saludables que contribuyan al aprendizaje por medio de promover un estado físico y mente saludable.

Notas

(1) Cuadros 2 y 3 recuperado de https:// www.stanfordchildrens.org/es/topic/ default?id=school-agedchildnutrition90-P05389

(2) seudónimo que reciben los ciudadanos de Costa Rica

\section{Referencias}

Austin, R. (2007) Super-Size Me and the Conundrum of Race/Ethnicity, Gender, and Class for

the Contemporary Law-Genre Documentary Filmmaker. Scholarship at Penn Law.

Blázquez, C. (2016) Revolución Industrial 4.0 cambiará no solo lo que hacemos, sino también lo que somos. CYC Prisma, 21; 10-25

Brody, J. (2017). El 'combustible' del aprendizaje: almuerzos nutritivos en las escuelas. The New York Times. (8 de junio de 2017). Recuperado de https://www.nytimes.com Cascales, M. (2008) Obesidad: pandemia del Siglo

XXI. Farmacia 11. Madrid 1-33. Recuperado de http://www.analesranf.com.

Costa-Font y Mas N (2014) ¿Globesity? The Effects of Globalization and Caloric IntakeCEsifo.

Working Paper Series, 4982.

Feuerbach, Ludwig (1850). Ensayo: "Enseñanza de la alimentación" (Lehre der Nahrungsmittel:

Für das Volk). Alemania
Hernández, D. y López, P. (2015) Hábitos de una alimentación adecuada en el proceso de

aprendizaje en el niño Kenneth de I nivel del Segundo Ciclo de Preescolar del Centro Educativo Alfonso Cortés del Distrito VI de Managua durante el II semestre del año 2015. Universidad Nacional Autónoma de Nicaragua; 1-71.

Jiménez C, Jennifer. (2019) “Costa Rica Vive la Peor Epidemia de Obesidad Registrada en toda

su Historia". Semanario Universidad, Universidad de Costa Rica (UCR) /San Pedro, Costa Rica/ 26 abr. Recuperado de https://www.ucr.ac.cr/noticias/2019

Ministerio de Educación y Equipo Técnico Nacional del Censo. (2017) Informe Ejecutivo. Censo

Escolar Peso/Talla Costa Rica. Ministerio de Educación Pública/Ministerio de Salud de Costa Rica,1; $1-12$.

Organización Mundial de la Salud (2004). Organización Mundial de la Salud. Estrategia mundial sobre régimen alimentario, actividad física y salud de la $57^{\text {a }}$ Asamblea Mundial de la Salud. Resolución WHO_57.17. Aprobación por los estados miembros de la Resolución WHO_55.23. Recuperado en 2010, de http://apps.who.int/gb/

Organización Mundial de la Salud. (2012). Estadísticas Sanitarias Mundiales una Instantánea de la Salud Mundial. Ginebra. Recuperado de www.who.int. Organización Mundial de la Salud. (2016). Informe de la Comisión para Acabar con la Obesidad Infantil. Ginebra. Recuperado de www.who.int. Organización Mundial de la Salud. Obesidad y sobrepeso (2017). Recuperado de http:/www.who.int/media centre/factsheets/fs311/es/ Pintor, E., Herreros, B., Gargantilla, P. y Vivas E., (2017) Super size me (2004): ¿La película que cambió McDonald's? Rev Med Cine [Internet], 13 (1); 3-8. 


\section{II: Salud, Educación y Medios de Comunicación}

Revista CyC Prisma. (2016) Revolución Industrial 4.0. Entrevista con Robert Shiller volumen 21 ; 6-15.

Recuperado de: http://revistaprismacyc.com/prismacyc21/index. html

Spurlock, M. (2004). Film: Super Size Me. (2004) Dir. Morgan. Samuel Goldwyn Films / Roadside Attractions / Showtime. Género: Documental Sátira/Cocina. DVD/VoD

Universidad de Stanford. "Nutrición: Edad Escolar". Sandford Children's Health. Publicación
Web. Recuperado de: https://www.stanfordchildrens.org/es/ topic/default?id=school-agedchildnutrition-90-P05389. 1 de setiembre del 2019

UNESCO Education. (2007) Una nutrición adecuada propicia el aprendizaje: la hora de la comida en un centro preescolar de Johannesburgo (Sudáfrica). Informe de Seguimiento de la Educación para todos en el Mundo; 114-125. Recuperado de: http:/www. unesco.org/education/GMR/2007/es/capitulo5.pdf 